\title{
Spike Timing and Information Transmission at Retinogeniculate Synapses
}

\author{
Daniel L. Rathbun, ${ }^{1,2}$ David K. Warland, ${ }^{3}$ and W. Martin Usrey ${ }^{1,3,4}$ \\ ${ }^{1}$ Center for Neuroscience, University of California, Davis, Davis, California 95618, ${ }^{2}$ Institute for Ophthalmology and Center for Integrative Neuroscience, \\ University of Tuebingen, D-72076 Tuebingen, Germany, ${ }^{3}$ Department of Neurobiology, Physiology, and Behavior, University of California, Davis, Davis, \\ California 95616, and ${ }^{4}$ Department of Neurology, University of California, Davis, Sacramento, California 95817
}

This study examines the rules governing the transfer of spikes between the retina and the lateral geniculate nucleus (LGN) with the goal of determining whether the most informative retinal spikes preferentially drive LGN responses and what role spike timing plays in the process. By recording from monosynaptically connected pairs of retinal ganglion cells and LGN neurons in vivo in the cat, we show that relayed spikes are more likely than nonrelayed spikes to be evoked by stimuli that match the receptive fields of the recorded cells and that an interspike interval-based mechanism contributes to the process. Relayed spikes are also more reliable in their timing and number where they often achieve the theoretical limit of minimum variance. As a result, relayed spikes carry more visual information per spike. Based on these results, we conclude that retinogeniculate processing increases sparseness in the neural code by selectively relaying the highest fidelity spikes to the visual cortex.

\section{Introduction}

Retinal ganglion cells produce many more spikes than their postsynaptic targets in the lateral geniculate nucleus (LGN) of the thalamus (Hubel and Wiesel, 1961; Cleland et al., 1971; Kaplan et al., 1987; Mastronarde, 1987; Usrey et al., 1998, 1999; Levine and Cleland, 2001; Rowe and Fischer, 2001; Sincich et al., 2007, 2009a; Weyand, 2007), yet it appears that little information loss occurs between the retina and the LGN (Sincich et al., 2009a). Because the LGN is the major source of visual information supplied to primary visual cortex, the recoding of visual information by LGN neurons has important ramifications for all downstream processing of events in the visual world. An important question we address here is whether retinal spikes carrying the most visual information are preferentially passed from the retina to the LGN and, if so, what role spike timing plays in this process.

The retinogeniculate pathway is an ideal substrate for studying the relationship between presynaptic and postsynaptic activity and the transmission of sensory information. Unlike cortical neurons, LGN neurons receive feedforward input from a very small number of retinal ganglion cells, typically one to five (Cleland, 1986; Reid and Usrey, 2004). Consequently, these inputs are generally quite strong and effective at driving suprathreshold responses. Indeed, simultaneous recordings of monosynaptically connected retinal ganglion cells and LGN neurons demonstrate

Received Feb. 18, 2010; revised July 7, 2010; accepted July 12, 2010

This work was supported by National Institutes of Health Grants EY013588, EY016182, and EY012576, and National Science Foundation Grant 0705427. We thank Katie Neverkovec, Kelly Henning, and Dan Sperka for technical support. Xin Wang, Judith Hirsch, and Fritz Sommer provided insightful comments on previous versions of this manuscript.

Correspondence should be addressed to W. Martin Usrey, Center for Neuroscience, University of California, Davis, 1544 Newton Court, Davis, CA 95618. E-mail: wmusrey@ucdavis.edu.

D01:10.1523/JNEUROSCI.0909-10.2010

Copyright $\odot 2010$ the authors $\quad 0270-6474 / 10 / 3013558-09 \$ 15.00 / 0$ that spikes from an individual retinal ganglion cell can reliably drive spikes in a postsynaptic LGN neuron (Cleland et al., 1971; Mastronarde, 1987; Usrey et al., 1998, 1999; Levine and Cleland, 2001; Rowe and Fischer, 2001; Weyand, 2007). Furthermore, results from studies examining S-potentials (an extracellular measure of the retinal EPSP) indicate that nearly every spike produced by an LGN neuron can be attributed to spikes arriving from the retina (Kaplan and Shapley, 1984; Sincich et al., 2007). With this in mind, there are also interesting dynamics influencing the transmission of spikes between the retina and LGN, as retinal spikes that occur after short interspike intervals (ISIs) $(<30 \mathrm{~ms})$ are more effective at evoking postsynaptic spikes than spikes that follow longer ISIs (Mastronarde, 1987; Usrey et al., 1998; Levine and Cleland, 2001; Rowe and Fischer, 2001; Sincich et al., 2007; Weyand, 2007). If this dynamic efficacy serves to capture those retinal spikes that are most informative about the external world (Rathbun et al., 2007; Sincich et al., 2009a; Uglesich et al., 2009), then retinal spikes relayed to cortex should have a stronger correlation with the visual stimulus than nonrelayed spikes. Relayed spikes should also have more information per spike than nonrelayed spikes. To test these hypotheses, we made simultaneous recordings from monosynaptically connected retinal ganglion cells and LGN neurons in the anesthetized cat and studied the visual properties of relayed and nonrelayed spikes. Compared with nonrelayed spikes, we found that relayed spikes were more likely to be evoked by stimuli that matched the receptive field of the cell. We also found that most cells used a neural code that was well described by sequences of firing events and that these cells operated near the fundamental physical limits of precision. Finally, the average information conveyed by a single spike increased across the retinogeniculate synapse. These results demonstrate that the LGN is much more than a simple relay for retinal signals to cortex; it is a structure that serves to improve the visual signal and increase overall coding efficiency. 


\section{Materials and Methods}

\section{Surgery and preparation}

All surgical and experimental procedures were performed with the approval of the Animal Care and Use Committee at the University of California, Davis. Adult cats, both sexes, were initially anesthetized with ketamine $(10 \mathrm{mg} / \mathrm{kg}, \mathrm{i} . \mathrm{m}$.) and maintained with thiopental sodium (10 $\mathrm{mg} / \mathrm{kg}$, i.v.). Wound margins were infused with lidocaine before and after each incision. Animals were placed in a stereotaxic apparatus and mechanically respired. Electrocardiogram, EEG, and expired $\mathrm{CO}_{2}$ were continuously monitored for the duration of the experiment. Anesthesia was maintained with thiopental sodium $\left(2 \mathrm{mg} \cdot \mathrm{kg}^{-1} \cdot \mathrm{h}^{-1}\right.$, i.v. $)$. If physiological monitoring indicated a decrease in the level of anesthesia, additional thiopental was given and the rate of infusion increased.

A small craniotomy was made above the LGN, the dura was reflected, and the craniotomy was filled with agarose. The lateral margin of each eye was dissected and each sclera was glued to a ring mounted on the stereotaxic frame. These rings minimized eye movements and facilitated the introduction of an intraocular guide tube.

Once all surgical procedures were complete, the animal was paralyzed with vecuronium bromide $\left(0.2 \mathrm{mg} \cdot \mathrm{kg}^{-1} \cdot \mathrm{h}^{-1}\right.$, i.v. $)$. The pupils were dilated with $1 \%$ atropine sulfate and the nictitating membranes were retracted with $10 \%$ phenylephrine. Flurbiprofen sodium $(1.5 \mathrm{mg} / \mathrm{h})$ was administered to ensure pupillary dilation. The eyes were fitted with contact lenses and focused on a monitor located $1 \mathrm{~m}$ in front of the animal.

\section{Electrophysiological recording and visual stimuli}

Single-unit recordings were made simultaneously from the retina and LGN. The LGN recordings were made using a seven-channel multielectrode array (Thomas Recording). Retinal recordings were made using a single tungsten-in-glass microelectrode. Retinal recordings were made from regions of the right or left eye that projected to LGN layer A in the opposite hemisphere. The retinal electrode was introduced into the posterior chamber of the eye through a guide tube and positioned using a custom-made manipulator. Using a small spot of light and an audiomonitor, the region of the retina that evoked responses from the recordings in the LGN was determined and then targeted for retinal recordings. Neural responses were amplified, filtered, and recorded to a PC with a Power 1401 data acquisition interface and the Spike 2 software package (Cambridge Electronic Design). The spikes of individual neurons were isolated using template matching, parametric clustering, and the presence of a refractory period in the autocorrelogram.

Visual stimuli were created with a VSG 2/5 visual stimulus generator (Cambridge Research Systems) and presented on a gamma-calibrated Sony monitor with mean luminance of $38 \mathrm{~cd} / \mathrm{m}^{2}$. Receptive fields were mapped using a binary white-noise stimulus that consisted of a $16 \times 16$ grid of squares (Reid et al., 1997). Each square flickered independently between black and white according to an " $\mathrm{m}$-sequence." The monitor ran at a frame rate of $140 \mathrm{~Hz}$ and stimuli were updated with each monitor frame for $2^{15}-1$ frames ( $\sim 4 \mathrm{~min}$ ). Approximately $4-16$ squares of the stimulus overlapped the receptive field center of each neuron. A subset of neurons was also studied using a repeating, $10 \mathrm{~s}$ segment of the whitenoise stimulus presented in one or more blocks of 100 repetitions.

\section{Data analysis}

Cross-correlation analysis. Cross-correlation analysis was used to assess connectivity between neurons recorded from simultaneously in the retina and LGN during white-noise stimulation. Using a bin size of $0.1 \mathrm{~ms}$, an abrupt, short-latency peak in the cross-correlogram was taken as evidence of a monosynaptic connection between cell pairs (see Fig. 1) (Mastronarde, 1987; Usrey et al., 1998, 1999). Once the bin containing the peak was identified, all neighboring bins with values $>3$ SDs above the baseline mean were considered part of the peak. The baseline mean was calculated using bins from 30 to $50 \mathrm{~ms}$ on either side of the peak bin. Because each count in the cross-correlogram peak represents a single retinal spike relayed by the LGN neuron to cortex, these retinal spikes were termed "relayed" spikes, whereas the remaining retinal spikes were termed "nonrelayed." "Efficacy" was calculated as the percentage of retinal spikes relayed by the LGN neuron (Levick et al., 1972; Usrey et al., 1998, 1999).
Reverse-correlation analysis. Spatiotemporal receptive field (STRF) maps were generated from neuronal responses to a white-noise stimulus using reverse-correlation analysis. When receptive field maps were calculated separately for relayed and nonrelayed spikes, a randomly selected subset of spikes from the group with the most spikes was used to ensure the analysis was performed using spike-count-matched data sets (Rathbun et al., 2007). Since noise in the receptive field map typically decreases as more spikes are included in the reversecorrelation analysis, spike-count matching ensured that receptive field map differences were not influenced by spike number.

Maps were fitted with a spatiotemporal receptive field model to achieve smooth functions from which several parameters could be easily measured. Center $\left(F_{\mathrm{C}}\right)$ and surround $\left(F_{\mathrm{S}}\right)$ subregions were each treated as space/time separable sensitivity profiles $\left(F=S^{\star} T\right)$ in which the spatial component $(S)$ was a circularly symmetric Gaussian as follows:

$$
S(x, y)=A \cdot e^{\left(-\frac{x^{2}+y^{2}}{2 \sigma^{2}}\right)},
$$

and the temporal component $\left(T=T_{\mathrm{s}} \cdot T_{\mathrm{d}} \cdot T_{\mathrm{r}}\right)$ was the product of a sinusoid

$$
T_{\mathrm{s}}(t)=\cos \left[\frac{2 \pi(t-\delta)}{\tau_{\mathrm{s}}}\right],
$$

an exponential decay

$$
T_{\mathrm{d}}(t)=e^{\left[-\left(\frac{(t-\delta)}{\tau_{\mathrm{d}}}\right)^{2}\right]},
$$

and a sigmoid-shaped onset

$$
T_{\mathrm{r}}(t)=\frac{\left[(t-\delta) / \tau_{\mathrm{r}}\right]^{3}}{1+\left[(t-\delta) / \tau_{\mathrm{r}}\right]^{3}}
$$

(Rodieck, 1965; Schnapf et al., 1990). In this model, amplitude ( $A$ ) represents a relative scaling factor, $\sigma$ reflects the SD of the circularly symmetric Gaussian, $\delta$ is the stimulus-response delay, and $\tau_{\mathrm{s}}, \tau_{\mathrm{d}}$, and $\tau_{\mathrm{r}}$ are time constants for the sinusoid, exponential decay, and sigmoid rising phase, respectively. A constrained nonlinear optimization procedure (Matlab function: fmincon; The Mathworks) was used to fit these parameters to the full white-noise receptive field map. Finally, the full space/ time fit is given by the sum of the center and surround subregions as follows:

$$
\left(F_{\mathrm{ST}}=F_{\mathrm{C}}-F_{\mathrm{S}}\right) \text {. }
$$

To facilitate calculation of the normalized dot product (NDP), the continuous function of the STRF fit was calculated at each of the $16 \times 16 \times$ 16 points in space and time as the original STRF map.

A NDP was used to quantify the degree of similarity between the series of stimulus frames preceding each epoch of time (matching in length the number of frames associated with the STRF) and the fit of the STRF map (supplemental Fig. 1, available at www.jneurosci.org as supplemental material). The NDP was calculated using the following equation:

$$
\mathrm{NDP}=\frac{\sum_{i} \operatorname{sgn}\left(A_{i} * B_{i}\right) *\left|A_{i}\right|}{\sum_{i}\left|A_{i}\right|},
$$

where $i$ represents the $i$ th point in the $16 \times 16 \times 16$ STRF fit, $A$ represents the STRF vector, and $B$ represents the binary stimulus vector (with black squares represented by -1 and white squares represented by +1 ). Accordingly, the NDP is a single value, ranging from -1 to 1 , that provides a quantitative measure of how well the spatiotemporal stimulus at a given point in time matches the fitted spatiotemporal receptive field of a neuron, over all points $(i)$ in space and time. By calculating the full NDP series from the stimulus, we are, in essence, filtering the stimulus with the linear receptive field of the cell and expressing how well each point in the stimulus is expected to excite that neuron.

Analysis of spike train statistics. To quantify the number precision and timing precision of spike trains, we used the method described by Berry et 
al. (1997). Poststimulus time histograms (PSTHs) were created from retinal ganglion cell responses to a repeating $10 \mathrm{~s}$ clip of the white-noise stimulus (see Fig. 6). Separate PSTHs were made for (1) relayed spikes, (2) nonrelayed spikes, and (3) all retinal spikes (relayed plus nonrelayed). A relatively fine bin width of $0.3 \mathrm{~ms}$ was used to generate PSTHs because the data contained a high degree of temporal precision. Before identifying the event boundaries, we smoothed each PSTH with a Gaussian filter of width equal to the average jitter in the firing rate across all cells ( $\sigma f$ $=0.72 \mathrm{~ms}$ ). The width $\sigma f$ was determined by fitting a Gaussian curve to the spike train autocorrelation function for each cell and then averaging this value over all cells. Note that, once event boundaries were determined, the number and timing precision values were measured directly from spike times and no longer depended on the choice of smoothing. Event boundaries were identified as local minima $v$ that were significantly lower than neighboring maxima $p_{1}$ and $p_{2}$ at the $95 \%$ confidence level, such that $\sqrt{p_{1} p_{2}} / v \geq \varphi$, where the threshold $\varphi$ was chosen as 1.5. Each event in the PSTH was fitted with a normal distribution whose variance was taken as the spike timing variance of that event.

Information rates were calculated using the methods of Strong and colleagues (1998). Briefly, spike trains were discretized and converted to binary strings. These strings were broken into sets of "spike words" using a range of temporal bin sizes $(\Delta \tau)$ and word lengths $(T)$, where $\tilde{P}_{i}$ represents the normalized count of the $i$ th word. For any given combination of $\Delta \tau$ and $T$, entropy is estimated as follows:

$$
S(T, \Delta t ; \text { size })=-\sum_{i} \tilde{P}_{i} \log _{2} \tilde{P}_{i} .
$$

This notation is used to specifically emphasize that the estimate is dependent on data set size.

To correct for finite data set size, total and noise entropy were estimated by extrapolating true entropy $\left(S_{0}\right)$ from increasing fractions of the total data set using a least-squares fit to the form $S=S_{0}+S_{1} /$ size + $S_{2} /$ size $^{2}$. As spike words become long, entropy rate estimates become biased because of the finite available data. Therefore, moderately long word lengths were used to extrapolate entropy rates for arbitrarily long spike words at each $\Delta \tau$. Finally, coding efficiency was used to identify the physiologically relevant $\Delta \tau$ for each cell. The results reported were statistically significant for all $\Delta \tau$.

General statistics. Unless otherwise indicated, population data are summarized by the mean and SEM. Wilcoxon's signed-rank test was used to determine $p$ values for all pairwise statistical tests.

\section{Results}

To determine the influence of spike timing on visual processing and the transmission of information between the retina and LGN, we made simultaneous extracellular recordings from 17 pairs of monosynaptically connected retinal ganglion cells and LGN neurons in the anesthetized cat. For each pair of cells, spatiotemporal receptive field maps were made using a binary white-noise stimulus and reverse-correlation analysis (see Materials and Methods). Connectivity was independently assessed by performing a cross-correlation analysis on the simultaneously recorded spike trains (see Materials and Methods). Figure 1 shows the receptive field maps and cross-correlograms from two representative pairs
Cross Correlograms

Pair 1
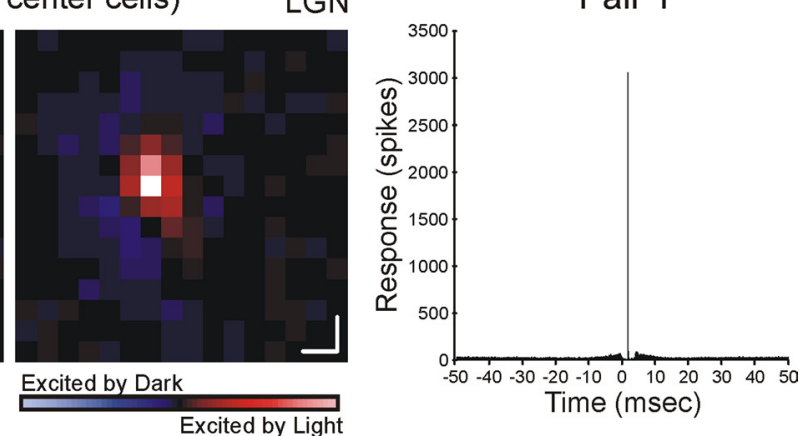

Pair 2
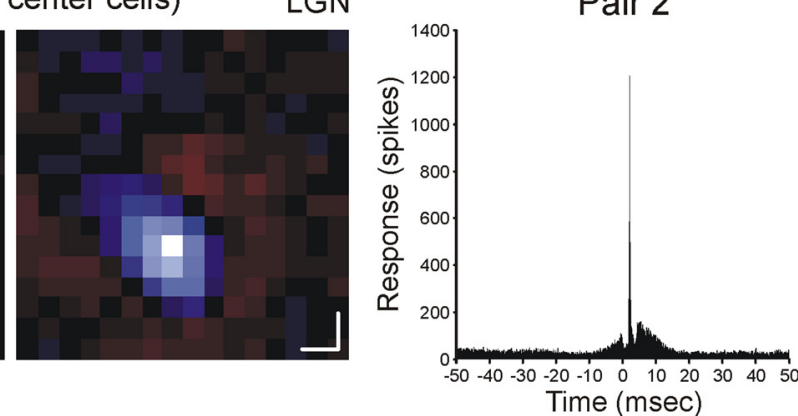

Time (msec)

Figure 1. Receptive fields and cross-correlograms from two pairs of cells — a retinal ganglion cell and an LGN neuron — that met the criteria for a monosynaptic connection. Each pair of cells was recorded from simultaneously, in vivo, with separate (he LN. Cells were excited with a spatiotemporal white-noise stimulus, and receptive fields were 列 show the relative activity of the LGN neuron. The abrupt, short latency peak in each cross-correlogram indicates that the LGN neuron often fired a spike in response to a retinal spike.

of cells ( 1 on-center pair, 1 off-center pair). In each correlogram, retinal spikes are set to time 0 and the abrupt and narrow peak to the right of zero $(\sim 2.5 \mathrm{~ms}$ latency) indicates that the LGN cell often fired a spike in response to the retinal input. Importantly, only a subset of retinal spikes evoked LGN spikes in their target neurons (34.6 and 13.8\% for pairs 1 and 2). Likewise, not all LGN spikes were evoked by spikes from their simultaneously recorded retinal input (38.0 and 52.6\% for pairs 1 and 2) (supplemental Fig. 2, available at www.jneurosci.org as supplemental material). Consistent with previous reports (Cleland et al., 1971; Mastronarde, 1987; Usrey et al., 1999), all of the monosynaptically connected pairs of cells in this study had overlapping receptive fields (6 on-center pairs, 10 off-center pairs, 1 on/off pair) (supplemental Fig. 2, available at www.jneurosci.org as supplemental material). None of the results reported below differed significantly for on versus off cell pairs. Because most of the cell pairs were composed of Y-cells (13 of 17 pairs; only 1 pair confidently identified as an $\mathrm{X}$-cell pair), it was not possible to examine differences between $\mathrm{X}$ - and Y-cells.

\section{Stimulus properties encoded by relayed spikes}

To address the question of whether retinal spikes that directly drive geniculate spikes differ from those that do not drive spikes, we used the cross-correlogram of each cell pair to sort retinal spikes into two groups: relayed spikes and nonrelayed spikes (see 


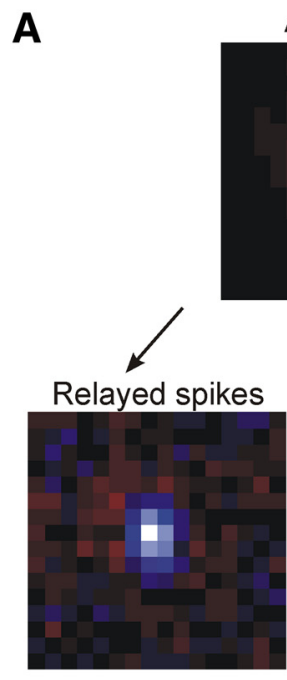

All spikes

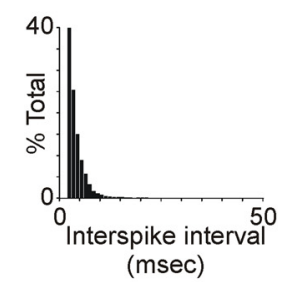

(msec)

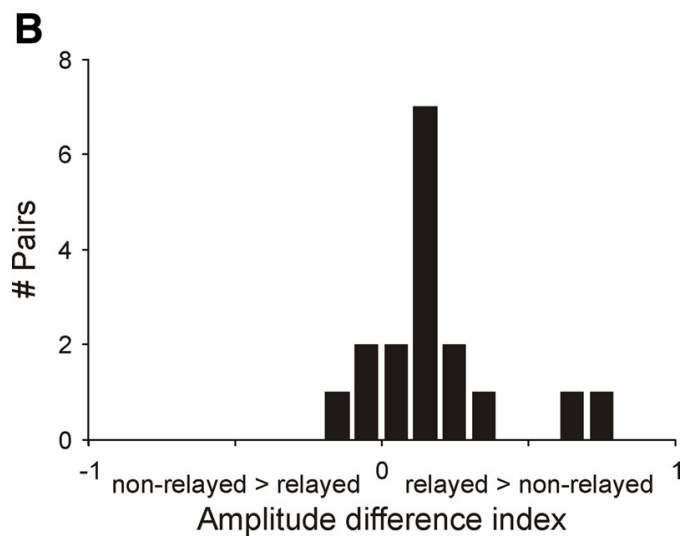

Figure 2. Relayed retinal spikes have a stronger correlation with a visual stimulus than nonrelayed spikes. $\boldsymbol{A}$, Three receptive field response maps made using three categories of spikes from a representative retinal ganglion cell: "all" spikes, "relayed" spikes, and "nonrelayed" spikes. Response maps were made using an equal number of spikes, and the maps are shown with the same color scale (pixel brightness) to compare response amplitude (correlation between stimulus and response) across the three categories of spikes. The histograms shown below the receptive field maps show the distribution of preceding ISIs for each category of spikes. The distribution of ISIs for relayed spikes is shifted to the left of that for nonrelayed spikes, indicating that relayed spikes are more likely to occur after short ISIs. B, Difference index histogram showing the relationship between the amplitudes of center subregions calculated from relayed and nonrelayed spikes across cell pairs ( $n=17$ pairs). The difference index is calculated as the difference between these amplitude values for relayed and nonrelayed spikes divided by their sum. Positive values indicate greater center amplitude for relayed spikes, and negative values indicate greater center amplitude for nonrelayed spikes.

Materials and Methods). We then used reverse-correlation analysis to generate separate receptive field maps for the relayed and nonrelayed spikes. As shown with an individual example in Figure $2 A$, the amplitude (indicated in pixel brightness) of the response map to stimuli in the receptive field center was greater for relayed spikes than for nonrelayed spikes. It should be noted that this analysis was done using a spike-count-matched data set to allow for direct comparison of response maps and to match noise levels in the measurements (Rathbun et al., 2007) (see Materials and Methods).

To compare the relative strength of center amplitudes across cell pairs, we used an amplitude index whereby positive values correspond to cases in which center amplitude was greater for relayed spikes and negative values correspond to cases in which center amplitude was greater for nonrelayed spikes. As shown in Figure $2 B$, index values are significantly greater than zero, on average, across our sample of cell pairs $(n=17 ; p<0.01)$, indicating that relayed spikes are more frequently than nonrelayed spikes to be driven by the visual stimulus.

To quantify the relationship between visual stimuli and relayed versus nonrelayed spikes further, we determined how well the white-noise stimulus that preceded each relayed spike matched the full receptive field of the retinal ganglion cell and compared these values to those for nonrelayed spikes. This process was also performed using the full receptive field of the connected LGN neuron. For this analysis, we separately calculated the NDP between the white-noise stimulus and the spatiotemporal receptive field fits for the retinal ganglion cell and the connected LGN neuron (see Materials and Methods) (supplemental Fig. 1, available at www.jneurosci.org as supplemental material). Using this approach, a NDP value of zero would indicate a random relationship between stimulus pixels and the receptive field of interest, a value of 1 would indicate that each pixel of the stimulus matched the receptive field in sign, and a value of -1 would indicate an inverted stimulus.

Using all frames of the full stimulus set, NDP values were normally distributed with a peak at 0 and a variance of 0.11 . More importantly, NDP values calculated using only those stimulus frames that preceded relayed spikes were significantly greater, on average, than those calculated using frames that preceded nonrelayed spikes (Fig. $3 A-C)(p<0.01)$. Indeed, NDP values were 1.37 times greater, on average across cell pairs, for relayed spikes compared with nonrelayed spikes when using the receptive field of the retinal ganglion cell and 2.28 times greater when using the receptive field of the LGN neuron. These results further demonstrate that relayed spikes follow stimuli that more closely match the receptive field of the retinal ganglion cell. Moreover, the finding that the relative increase in the NDP is greatest when using the receptive field of the LGN neuron is consistent with results from several studies demonstrating spatial and temporal differences in the receptive fields of synaptically connected retinal ganglion cells and LGN neurons-differences that include partially overlapping receptive fields, increased surround antagonism, and increased response transience in the LGN (Hubel and Wiesel, 1961; Cleland et al., 1971; Kaplan et al., 1987; Mastronarde, 1987; Mukherjee and Kaplan, 1995; Usrey et al., 1999). The finding that the relative increase in the NDP is greatest when using the receptive field of the LGN neuron is also consistent with the view that relayed spikes are likely to occur when evoked by stimuli that evoke responses from other (convergent) retinal ganglion cells with partially overlapping receptive fields that together establish the spatial organization of the LGN receptive field (Cleland, 1986; Mastronarde, 1989; Alonso et al., 2006; Chatterjee et al., 2007; Shlens et al., 2008).

We next examined the relationship between the NDP and the ISI that preceded each retinal spike. Several studies have shown that retinal spikes that follow short ISIs are more effective at driving LGN responses than spikes that follow longer ISIs (Mastronarde, 1987; Usrey et al., 1998; Levine and Cleland, 2001; Rowe and Fischer, 2001; Sincich et al., 2007; Weyand, 2007). This paired-spike enhancement is greatest for retinal 
A

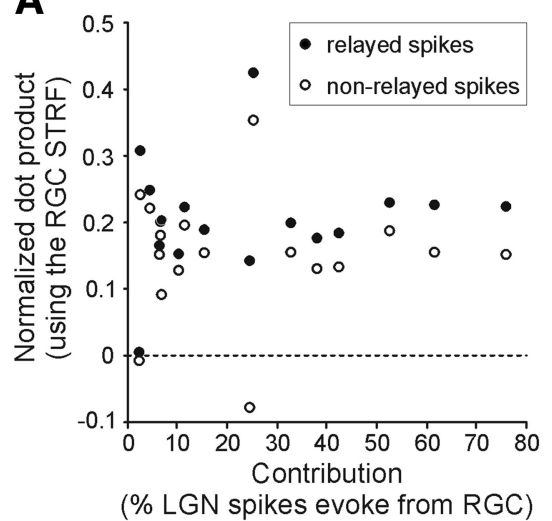

(\% LGN spikes evoke from RGC)

D

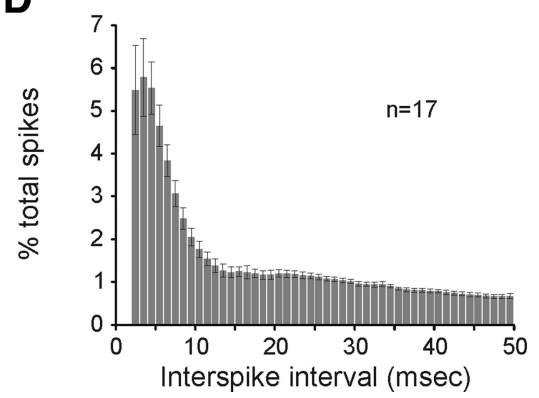

B

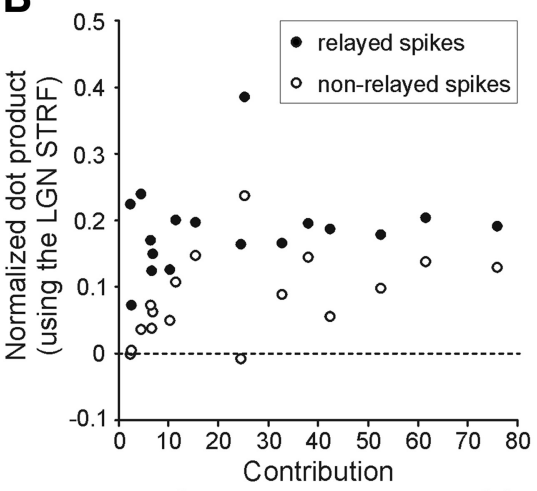

(\% LGN spikes evoke from RGC)

E

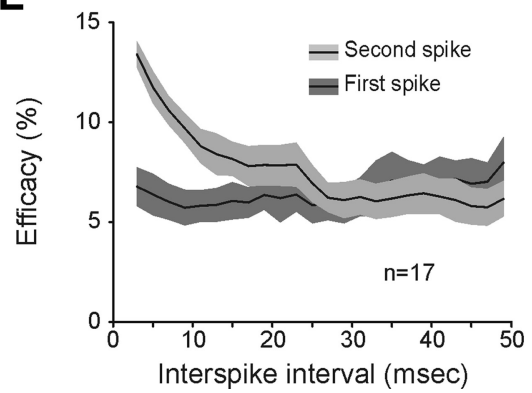

C

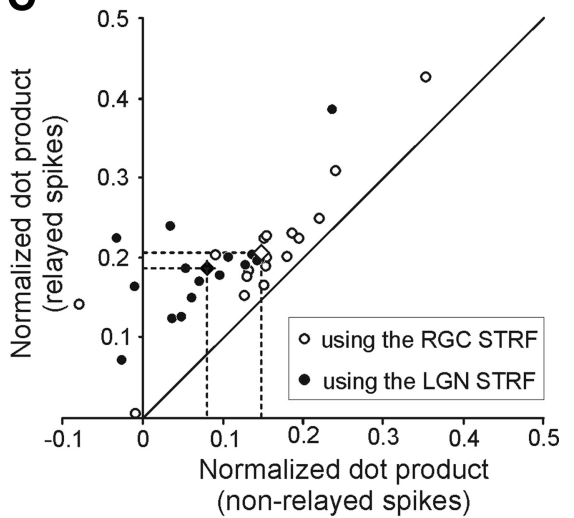

$\mathbf{F}$

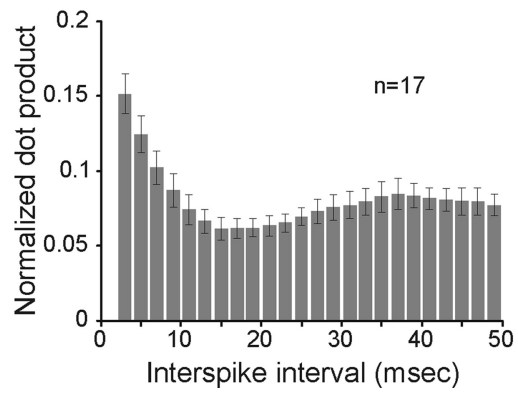

Figure 3. Visual stimuli that best match the receptive fields of the retinal ganglion cell and LGN neuron are more likely to evoke relayed spikes and are more likely to drive short ISI responses. $A$, Scatterplot showing the relationship between the visual stimulus preceding relayed and nonrelayed retinal spikes and the STRF map of the retinal ganglion cell— quantified with the NDP—as a function of the strength of connection, "contribution," between the retina and LGN. Contribution is equal to the percentage of LGN spikes that were evoked from the recorded retinal ganglion cell. Data points in vertical register (relayed and nonrelayed) are from the same pair of cells. Mean NDP values: Relayed spikes, $0.21 \pm 0.2$; nonrelayed spikes, $0.15 \pm 0.2$. B, Scatterplot showing the relationship between the visual stimulus preceding relayed and nonrelayed spikes and the STRF map of the LGN neuron — quantified with the NDP—as a function of the strength of connection, "contribution," between the retina and LGN. Mean NDP values: relayed spikes $=0.19 \pm 0.2$, nonrelayed spikes $=0.08 \pm 0.2$. C, Scatterplot showing the relationship—quantified with the NDP — between the visual stimulus and the STRF map of a cell for relayed and nonrelayed retinal spikes. The open symbols indicate values when using the STRF of the retinal ganglion cell, and the filled symbols indicate values when using the STRF of the LGN cell. When using either the retinal or LGNSTRF, NDP values are significantly greater for relayed spikes compared with nonrelayed spikes. $\boldsymbol{D}$, Mean ISI distribution from our sample of retinal ganglion cells. This distribution was calculated by averaging the normalized histograms for each retinal cell. Error bars indicate SEM. $\boldsymbol{E}$, Relationship between the efficacy of first and second retinal spikes in a pair as a function of ISI. Efficacy is the percentage of retinal spikes that drive LGN spikes. Second spikes have a greater efficacy than first spikes for ISIs up to $\sim 30 \mathrm{~ms}$. The gray bands denote SEM. $\boldsymbol{F}$, Histogram showing the mean relationship between normalized dot product values and retinal spikes that follow different ISIs, where the normalized dot product is a measure of how well the white-noise stimulus that preceded each spike matched the spatiotemporal receptive field map of each cell (see Materials and Methods). Retinal spikes that follow short ISIs are more likely to be evoked by stimuli that best match the receptive field of the retinal ganglion cell. Error bars indicate SEM.

spikes with ISIs just greater than the refractory period of the retinal neuron and continues for ISIs up to $\sim 30 \mathrm{~ms}$, where the first and second spike of a pair have equal probability of evoking a spike in the LGN. Across our sample of retinal ganglion cells, which had a median ISI of $16.9 \mathrm{~ms}$, paired-spike enhancement was robust and similar in shape to previous reports (Fig. $3 B, C$ ) (also see the ISI distributions in Fig. 2A). More importantly, NDP values calculated using the white-noise stimulus and the spatiotemporal receptive field fit for each individual retinal ganglion cell were greatest for retinal spikes after short ISIs (Fig. 3D). This finding indicates that the biophysical properties dictating synaptic efficacy and temporal summation are tuned to capture those retinal spikes that are driven by stimuli that best match the receptive field filter of the retinal ganglion cell.

An interesting prediction based on the ISI-dependent filter for retinogeniculate communication is an increased visual response latency for relayed versus nonrelayed retinal spikes. This prediction is based on the notion that the first retinal spike in a response is less likely to drive an LGN response than subsequent spikes in the response because of its longer preceding ISI. To test this prediction, we examined the temporal kernel corresponding to the receptive field center of retinal ganglion cells and compared the time to peak response for relayed and nonrelayed spikes. As

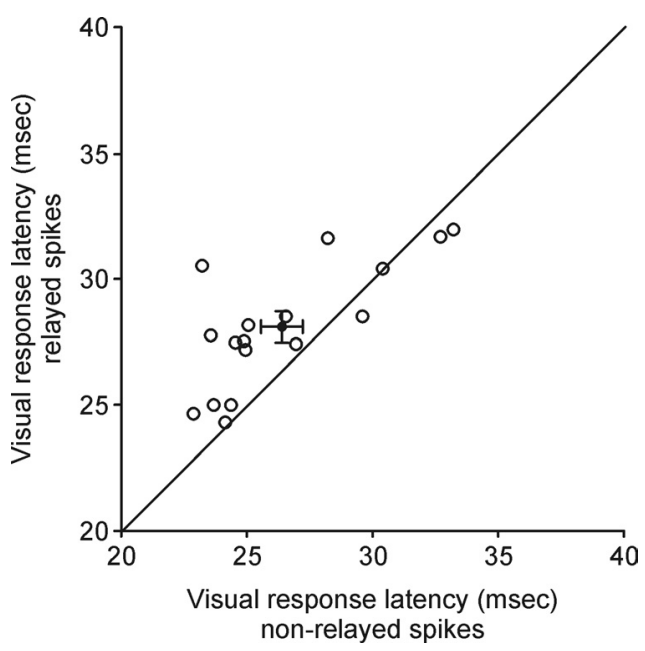

Figure 4. Scatterplot showing an increased latency between stimulus and response for relayed retinal spikes compared with nonrelayed spikes. Latency is taken from the impulse response of each spike class and corresponds to the time between stimulus and maximum response. Across our sample of cell pairs, relayed spikes have a significantly longer latency than nonrelayed spikes. The crosshairs indicate mean and SEM. 
A

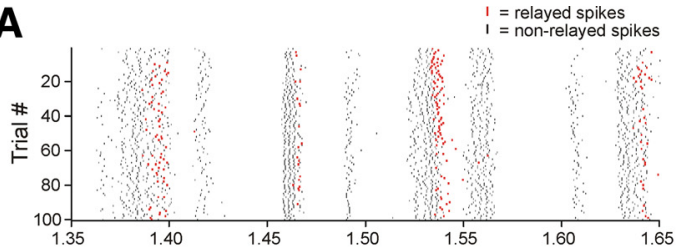

B All Retinal Spikes

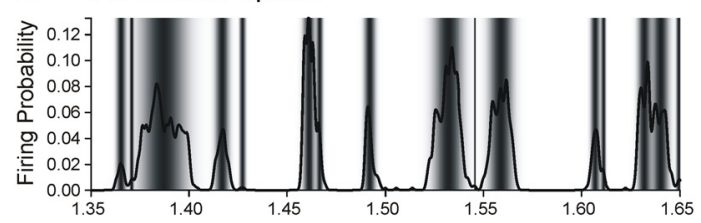

D Relayed Spikes

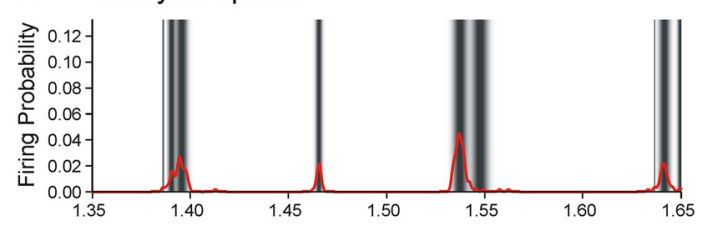

F Non-relayed Spikes

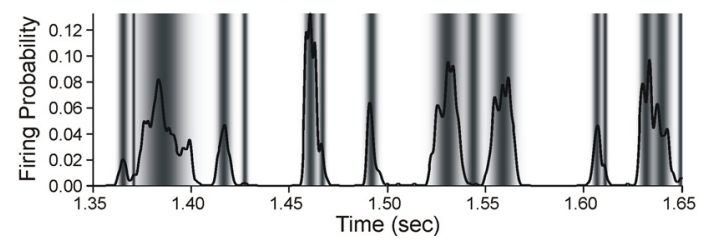

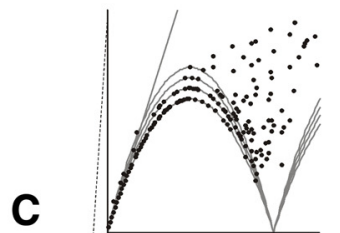

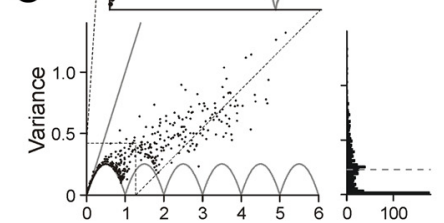

E

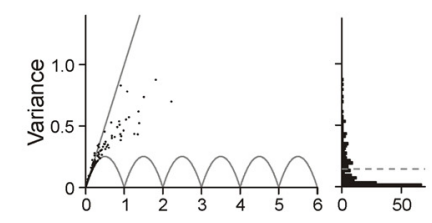

G

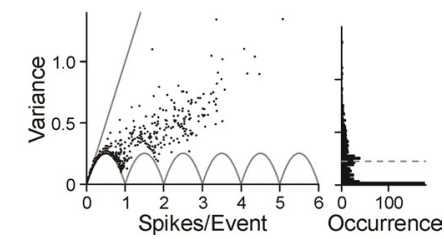

tively). These PSTHs were used to define event boundaries for subsequent analysis (see Materials and Methods). Figure 5, C, $E$, and $G$, shows the relationship between mean spike count and spike count variability for all of the events in the retinal spike train, the relayed events and the nonrelayed events. Consistent with previous findings (Kara et al., 2000), there was an overall increase in the Fano factor between the retina and LGN (supplemental Fig. 3, available at www.jneurosci.org as supplemental material). It is noteworthy that nearly all of the cells in our sample had sub-Poisson statistics, and that these spike trains often demonstrated a coding precision that approached the fundamental mathematical limits set by spike train statistics. The lower scallop-shaped gray line in Figure 5, $C, E$, and $G$, represents the theoretical minimum variance $\sigma^{2}=f(1-f)$ (where $f$ is the fractional mean spike count and $\sigma^{2}$ is the variance), and the upper straight line is the expected behavior if the neuron was described by a firing probability that varies with the stimulus (Poisson statistics). Similar to measurements in other visual systems, these neurons appear to use discrete firing events as the symbols with which to encode the visual world (Berry et al., 1997). Figure 5C, inset, shows how nested scallops are traced out by the data with each sequential scallop in this inset indicating one more deviation from the mathematical limit than the preceding scallop. Notably, since the majority of the firing events have a variance $<0.25$ spikes, firing events differing by even a single spike can be distinguished by downstream processing. Clearly, it appears that the responses of retinal ganglion cells are not well described by a firing rate alone.

shown in Figure 4, there was a modest, but significant, increase in the visual response latency for relayed spikes compared with nonrelayed spikes (mean latency difference, $1.7 \mathrm{~ms} ; p<0.01$ ). This finding indicates that retinogeniculate processing improves coding fidelity of the transmitted signal at the cost of modestly increasing response latency.

\section{Number precision and timing precision across the retinogeniculate synapse}

To study the influence of retinogeniculate processing on the number precision and timing precision of signals passed on to cortex, we analyzed the spike trains of monosynaptically connected retinal ganglion cells and LGN neurons to many repeats of a $10 \mathrm{~s}$ segment of the white-noise stimulus. Figure $5 \mathrm{~A}$ shows the spiking activity of a representative retinal ganglion cell to 100 repeats of the stimulus (only a $300 \mathrm{~ms}$ segment is shown to distinguish individual ticks); the black ticks correspond to nonrelayed spikes, and the red ticks correspond to relayed spikes. The PSTHs shown below Figure $5 A$ were generated separately using (1) both the relayed and nonrelayed spikes, (2) only the relayed spikes, and (3) only the nonrelayed spikes (Fig. $5 B, D, F$, respec-
An examination of event timing variance showed that relayed spikes have greater spike timing precision than nonrelayed spikes (Fig. 6A) ( $p<0.05)$ (see Materials and Methods). Likewise, relayed spikes had more spike number precision than nonrelayed spikes (Fig. $6 B)(p<0.01)$. For a final analysis, we examined the information content of each spike class (Fig. 6C). As expected from the spike number and spike timing differences between relayed and nonrelayed spikes, relayed spikes were found to transmit more visual information per spike than nonrelayed spikes $(p<0.01)$. Consistent with the theory of sparse coding (Olshausen and Field, 2004), these results demonstrate that the processing of spikes at the retinogeniculate synapse serves to recode the visual signal with the same fidelity using a smaller number of spikes, thereby improving the overall coding efficiency of the visual system.

\section{Discussion}

Visual signals leaving the eye do not have direct access to the cortex, but rather must be relayed to the cortex by neurons in the LGN. Because retinal ganglion cells produce many more spikes than their postsynaptic targets in the LGN, only a fraction of the 


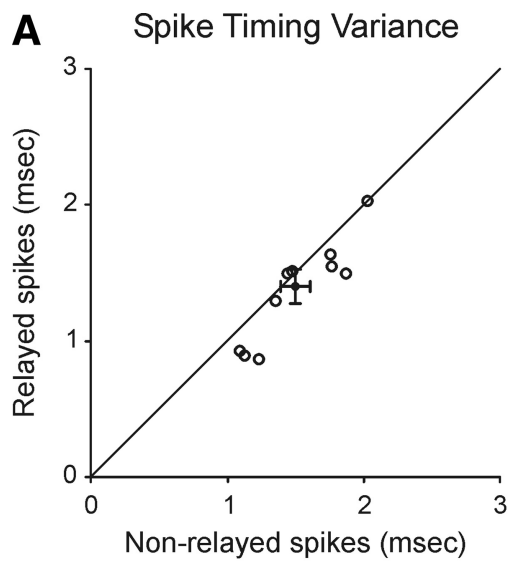

\section{B Spike Number Variance}
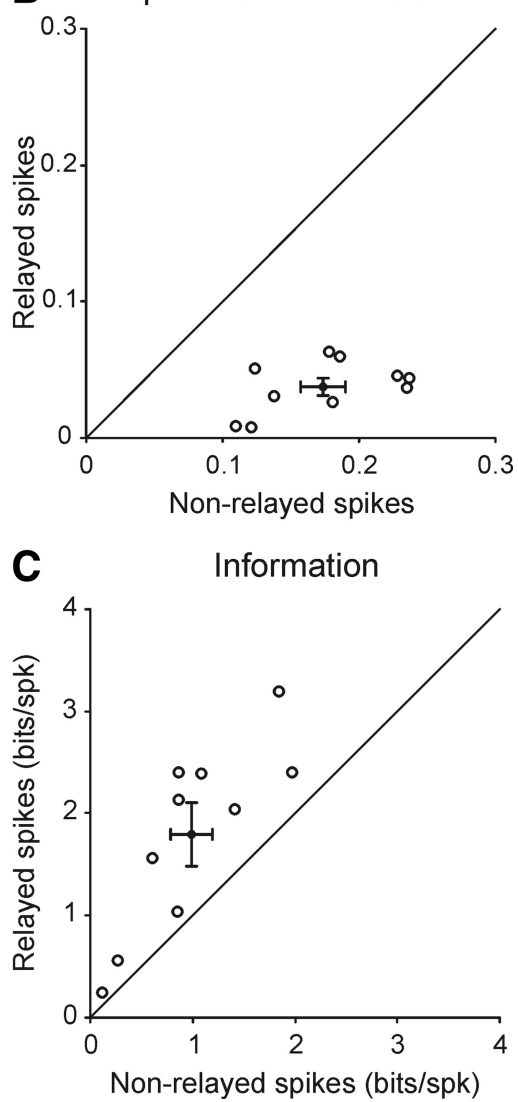

Figure 6. Spike timing and spike number are more precise for relayed spikes compared with nonrelayed spikes, leading to higher information content. $A$, Scatterplot comparing the temporal variance of relayed and nonrelayed spikes. Relayed spikes occur with significantly greater spike timing precision (less variance) than nonrelayed spikes. $\boldsymbol{B}$, Scatterplot comparing the spike number variance of relayed and nonrelayed spikes. Relayed spikes have significantly less variance in spike number than nonrelayed spikes. C, Scatterplot comparing the amount of information (bits/spike) contained in relayed and nonrelayed spikes. Relayed spikes carry significantly greater information than nonrelayed spikes. The crosshairs indicate mean and SEM.

spikes generated in the retina reach the cortex. The goal of this study was to determine how this filtering of spikes by LGN neurons affects the visual signal that reaches the cortex. In particular, we wanted to know whether the most informative retinal spikes preferentially drive LGN responses, how relayed and nonrelayed spikes differ in their response characteristics, and whether spike timing plays a role in the selection process. By recording simultaneously the spiking activity of monosynaptically connected pairs of retinal ganglion cells and LGN neurons, we compared the visual responses of relayed and nonrelayed retinal spikes. Unlike S-potential recordings that allow for a comparison between the spike trains of an LGN neuron and its strongest retinal input, paired-cell recordings allow for a comparison between the spike trains of an LGN neuron and any of its retinal inputs, strong or weak. For all pairs of cells, with strong or weak connections, our results show that relayed spikes are more likely than nonrelayed spikes to be evoked by stimuli that match the receptive field of a cell. An ISI-based mechanism contributes to this phenomenon, as retinal spikes that occur after a short ISI are not only more likely to drive a postsynaptic spike but also more frequently evoked by stimuli that best match the receptive field of the cell. In addition, relayed spiking events were more precise in spike timing and spike count. Consequently, relayed spikes carry more information than nonrelayed spikes. In the sections below, we discuss the cellular mechanisms that govern retinogeniculate communication and consider the implications of our results for information processing in the early visual system with an emphasis placed on how spike processing in the LGN may increase coding efficiency through encoding the information more sparsely.

\section{Functional properties of retinogeniculate connections}

Across species, the receptive fields of LGN neurons closely resemble those of their retinal afferents, in terms of retinotopic location, receptive field size, and the spatial organization of on and off responses (Hubel and Wiesel, 1961; Cleland et al., 1971; Levick et al., 1972; Usrey and Reid, 1998, 1999; Sincich et al., 2007, 2009b; Wang et al., 2007). These similarities are likely the result of a precise organization of connections between the retina and LGN with minimal convergence. Indeed, individual LGN neurons in the cat typically receive convergent input from a small ensemble of retinal ganglion cells, typically one to five cells (for review, see Cleland, 1986; Reid and Usrey, 2004). An important consequence of this low convergence is that individual afferents are quite strong and capable of driving suprathreshold responses. Thus, unlike the cortex where large numbers of weak afferents must work together to bring a target cell to spike threshold, a single retinal afferent can reliably drive postsynaptic spikes in the LGN.

Unlike the subtle differences in spatial structure between the receptive fields of retinal ganglion cells and LGN neurons, there are dramatic differences in the temporal structure of their spike trains. As mentioned above, individual retinal ganglion cells provide strong input to target cells in the LGN; however, isolated retinal spikes rarely drive suprathreshold responses. Rather, retinal spikes are most likely to drive postsynaptic spikes when they occur shortly after a previous retinal spike (Mastronarde, 1987; Usrey et al., 1998; Levine and Cleland, 2001; Rowe and Fischer, 2001; Sincich et al., 2007; Weyand, 2007). This increased efficacy for driving a response is greatest for retinal spikes with the shortest preceding ISIs $(\sim 2 \mathrm{~ms}$, limited by the refractory period of the cell) and decreases with increasing ISI to $\sim 30 \mathrm{~ms}$, where the first and second spike in a pair have equally low efficacy at driving a postsynaptic spike (Fig. 3C). Although numerous presynaptic and postsynaptic mechanisms influence synaptic communication between retinal ganglion cells and LGN neurons (Chen et al., 2002; Blitz and Regehr, 2003; Chen and Regehr, 2003; Dan and Poo, 2006), temporal summation appears to make the greatest contribution to the enhanced efficacy of retinal spikes that follow short ISIs, as evidenced by a recent modeling paper demonstrating that the spike train of an LGN neuron can be accurately predicted from the retinal spike train by taking into account 
postsynaptic summation (Carandini et al., 2007). Interestingly, retinal spikes that precede a pair with a given ISI have a relatively minor influence on enhancing the efficacy of the second spike in the pair (Usrey et al., 1998). Thus, the memory of an LGN neuron appears to be rather short, with the single ISI that precedes any given spike having the most significant influence on the likelihood that the LGN neuron will reach threshold.

In layer 4 of primary visual cortex, an increased efficacy for short ISI LGN spikes occurs not only when the spikes establishing the ISI come from the same LGN neuron (homosynaptic interactions) but also when they come from two different LGN neurons providing convergent input (heterosynaptic interactions) (Usrey et al., 2000). Although the opportunities for heterosynaptic interactions should be less for LGN neurons than for cortical neurons (because of an approximate sixfold decrease in the number of convergent inputs) (Reid and Usrey, 2004), the basic mechanism of temporal summation should increase the efficacy of short ISI spikes when the spikes come from the same retinal axon or different retinal axons.

A major finding of this study is that relayed spikes are more likely than nonrelayed spikes to be evoked by stimuli that match the receptive field of a cell. This finding has a clear underlying cellular mechanism, as retinal spikes that follow short ISIs are more frequently evoked by stimuli that best match the receptive field of the cell (Figs. 2A, 3D) (Rathbun et al., 2007) and more likely to drive postsynaptic spikes in their target neurons via postsynaptic summation (Mastronarde, 1987; Usrey et al., 1998; Levine and Cleland, 2001; Rowe and Fischer, 2001; Carandini et al., 2007; Sincich et al., 2007; Weyand, 2007). Importantly, the enhanced efficacy of second spikes in a pair and the increased correlation of second spikes with the visual stimulus follow a similar ISI-dependent time course, indicating that the biophysical properties that govern postsynaptic summation are tuned to capture those retinal spikes evoked by stimuli with the greatest signal. An interesting question is whether this tuning can be adjusted by behavior or by altering the statistics of the visual environment. Evidence supporting these possibilities comes from attention studies that find increased activity among LGN cells when spatial attention is directed toward their receptive fields, as well as from experiments showing that membrane time constants are dynamic and can be increased or decreased by altering the overall activity of afferent inputs and by changing the amount of modulatory input a cell receives (Koch et al., 1996; Zhu and Uhlrich, 1997; Sanchez-Vives et al., 2000; Vanduffel et al., 2000; O'Connor et al., 2002; Uhlrich et al., 2002; Kastner et al., 2004; Alitto et al., 2005; McAlonan et al., 2008).

\section{Sparse coding}

The theoretical principles behind sparse coding have had broad impact in disciplines such as engineering and mathematics in addition to the fundamental insights they have given into the coding strategies used by the brain. At its core, sparse coding seeks to represent a signal using the smallest number of elements selected out of a large dictionary. In addition to the benefit of providing a compact representation of signals, sparse coding has found a wide range of applications in signal and image analysis tasks including denoising, superresolution, inpainting, sound source separation, extraction of key features for object recognition, and has provided the foundation for new fields such as compressed sensing.

In the field of neuroscience, it has been known for some time that many neurons in higher stages of sensory processing tend to fire less frequently than those at lower stages. This observation is often attributed to higher levels of the nervous system representing sensory information that is more specific than at lower levels (Barlow, 1972). It has been widely argued that such sparse representations are important for a number of biological reasons, including coding and energy efficiency, making the structure of natural signals more explicit, making the information easier to read out or process at higher stages of processing, and increasing the storage capacity of memory (Olshausen and Field, 2004). Recent studies showing that the amount of information available in the spike train of an LGN neuron is similar to that of its higher firing rate retinal afferent (Sincich et al., 2009a; Uglesich et al., 2009) further support the idea that the retinogeniculate synapse is re-representing the incoming retinal information in a sparse form. Although once considered a structure that only relayed spikes from the retina to the cortex, the results of this paper and other recent evidence demonstrate that the LGN is contributing significantly to shaping the visual signal before it arrives to cortex. Our finding that the average information conveyed by a single spike increases across the retinogeniculate synapse by selectively transmitting retinal spikes with the most information, supports the idea that the LGN uses the principles of sparse coding to improve downstream visual processing and coding efficiency.

\section{References}

Alitto HJ, Weyand TG, Usrey WM (2005) Distinct properties of stimulusevoked bursts in the lateral geniculate nucleus. J Neurosci 25:514-523.

Alonso JM, Yeh CI, Weng C, Stoelzel C (2006) Retinogeniculate connections: a balancing act between connection specificity and receptive field diversity. Prog Brain Res 154:3-13.

Barlow HB (1972) Single units and sensation: a neuron doctrine for perceptual psychology. Perception 1:371-394.

Berry MJ, Warland DK, Meister M (1997) The structure and precision of retinal spike trains. Proc Natl Acad Sci U S A 94:5411-5416.

Blitz DM, Regehr WG (2003) Retinogeniculate synaptic properties controlling spike number and timing in relay neurons. J Neurophysiol 90:2438-2450.

Carandini M, Horton JC, Sincich LC (2007) Thalamic filtering of retinal spike trains by postsynaptic summation. J Vis 7:20.1-11.

Chatterjee S, Merwine DK, Amthor FR, Grzywacz NM (2007) Properties of stimulus-dependent synchrony in retinal ganglion cells. Vis Neurosci 24:827-843.

Chen C, Regehr WG (2003) Presynaptic modulation of the retinogeniculate synapse. J Neurosci 23:3130-3135.

Chen C, Blitz DM, Regehr WG (2002) Contributions of receptor desensitization and saturation to plasticity at the retinogeniculate synapse. Neuron 33:779-788.

Cleland BG (1986) The dorsal lateral geniculate nucleus of the cat. In: Visual neuroscience (Pettigrew JD, Sanderson KS, Levick WR, eds), p 111. London: Cambridge UP.

Cleland BG, Dubin MW, Levick WR (1971) Simultaneous recording of input and output of lateral geniculate neurones. Nat New Biol 231:191-192.

Dan Y, Poo MM (2006) Spike timing-dependent plasticity: from synapse to perception. Physiol Rev 86:1033-1048.

Hubel DH, Wiesel TN (1961) Integrative action in the cat's lateral geniculate body. J Physiol 155:385-398.

Kaplan E, Shapley R (1984) The origin of the S (slow) potential in the mammalian lateral geniculate nucleus. Exp Brain Res 55:111-116.

Kaplan E, Purpura K, Shapley RM (1987) Contrast affects the transmission of visual information through the mammalian lateral geniculate nucleus. J Physiol 391:267-288.

Kara P, Reinagel P, Reid RC (2000) Low response variability in simultaneously recorded retinal, thalamic, and cortical neurons. Neuron 27:635-646.

Kastner S, O'Connor DH, Fukui MM, Fehd HM, Herwig U, Pinsk MA (2004) Functional imaging of the human lateral geniculate nucleus and pulvinar. J Neurophysiol 91:438-448.

Koch C, Rapp M, Segev I (1996) A brief history of time (constants). Cereb Cortex 6:93-101. 
Levick WR, Cleland BG, Dubin MW (1972) Lateral geniculate neurons of cat: retinal inputs and physiology. Invest Ophthalmol Vis Sci 11:302-311.

Levine MW, Cleland BG (2001) An analysis of the effect of retinal ganglion cell impulses upon the firing probability of neurons in the dorsal lateral geniculate nucleus of the cat. Brain Res 902:244-254.

Mastronarde DN (1987) Two classes of single-input X-cells in cat lateral geniculate nucleus. II. Retinal inputs and the generation of receptive-field properties. J Neurophysiol 57:381-413.

Mastronarde DN (1989) Correlated firing of retinal ganglion cells. Trends Neurosci 12:75-80.

McAlonan K, Cavanaugh J, Wurtz RH (2008) Guarding the gateway to cortex with attention in visual thalamus. Nature 456:391-394.

Mukherjee P, Kaplan E (1995) Dynamics of neurons in the cat lateral geniculate nucleus: in vivo electrophysiology and computation modeling. J Neurophysiol 74:1222-1243.

O'Connor DH, Fukui MM, Pinsk MA, Kastner S (2002) Attention modulates responses in the human lateral geniculate nucleus. Nat Neurosci 5:1203-1209.

Olshausen BA, Field DJ (2004) Sparse coding of sensory inputs. Curr Opin Neurobiol 14:481-487.

Rathbun DL, Alitto HJ, Weyand TG, Usrey WM (2007) Interspike interval analysis of retinal ganglion cell receptive fields. J Neurophysiol 98:911-919.

Reid RC, Usrey WM (2004) Functional connectivity in the pathway from retina to striate cortex. In: The visual neurosciences (Chalupa LM, Werner JS, eds), pp 673-679. Cambridge, MA: MIT.

Reid RC, Victor JD, Shapley RM (1997) The use of m-sequences in the analysis of visual neurons: linear receptive field properties. Vis Neurosci 14:1015-1027.

Rodieck RW (1965) Quantitative analysis of cat retinal ganglion cell response to visual stimuli. Vision Res 5:583-601.

Rowe MH, Fischer Q (2001) Dynamic properties of retino-geniculate synapses in the cat. Vis Neurosci 18:219-231.

Sanchez-Vives MV, Nowak LG, McCormick DA (2000) Membrane mechanisms underlying contrast adaptation in cat area 17 in vivo. J Neurosci 20:4267-4285.
Schnapf JL, Nunn BJ, Meister M, Baylor DA (1990) Visual transduction in cones of the monkey Macaca fascicularis. J Physiol 427:681-713.

Shlens J, Rieke F, Chichilnisky E (2008) Synchronized firing in the retina. Curr Opin Neurobiol 18:396-402.

Sincich LC, Adams DL, Economides JR, Horton JC (2007) Transmission of spike trains at the retinogeniculate synapse. J Neurosci 27:2683-2692.

Sincich LC, Horton JC, Sharpee TO (2009a) Preserving information in neural transmission. J Neurosci 29:6207-6216.

Sincich LC, Zhang Y, Tiruveedhula P, Horton JC, Roorda A (2009b) Resolving single cone inputs to visual receptive fields. Nat Neurosci 12:967-969.

Strong SP, Koberle R, de Ruyter van Steveninck RR, Bialek W (1998) Entropy and information in neural spike trains. Phys Rev Lett 80:197.

Uglesich R, Casti A, Hayot F, Kaplan E (2009) Stimulus size dependence of information transfer from retina to thalamus. Front Syst Neurosci 3:10.

Uhlrich DJ, Manning KA, Xue JT (2002) Effects of activation of the histaminergic tuberomammillary nucleus on visual responses of neurons in the dorsal lateral geniculate nucleus. J Neurosci 22:1098-1107.

Usrey WM, Reppas JB, Reid RC (1998) Paired-spike interactions and synaptic efficacy of retinal inputs to the thalamus. Nature 395:384-387.

Usrey WM, Reppas JB, Reid RC (1999) Specificity and strength of retinogeniculate connections. J Neurophysiol 82:3527-3540.

Usrey WM, Alonso JM, Reid RC (2000) Synaptic interactions between thalamic inputs to simple cells in cat visual cortex. J Neurosci 20:5461-5467.

Vanduffel W, Tootell RB, Orban GA (2000) Attention-dependent suppression of metabolic activity in the early stages of the macaque visual system. Cereb Cortex 10:109-126.

Wang X, Wei Y, Vaingankar V, Wang Q, Koepsell K, Sommer FT, Hirsch JA (2007) Feedforward excitation and inhibition evoke dual modes of firing in the cat's visual thalamus during naturalistic viewing. Neuron 55:465-478.

Weyand TG (2007) Retinogeniculate transmission in wakefulness. J Neurophysiol 98:769-785.

Zhu JJ, Uhlrich DJ (1997) Nicotinic receptor-mediated responses in relay cells and interneurons in the rat lateral geniculate nucleus. Neuroscience 80:191-202. 